\title{
MicroRNAs: A New Understanding of Platelet Physiology and Pathology
}

\author{
Christian Schulte ${ }^{1,2}$ Manuel Mayr ${ }^{1}$ \\ ${ }^{1}$ King's British Heart Foundation Centre, King's College London, \\ London, United Kingdom \\ 2 Department of General and Interventional Cardiology, University \\ Heart Centre Hamburg Eppendorf, Hamburg, Germany
}

Thromb Haemost 2019;119:191.

Platelets have roles in both health and disease beyond their role in haemostasis. As platelets are anucleate and do not perform transcription, micro-ribonucleic acids (miRNAs) are in the spotlight as post-transcriptional regulators of their protein content. Through discoveries that have partly emanated from our group, ${ }^{1,2}$ it has now become apparent that miRNAs are relevant to platelet biology. Using human megakaryocytes in addition to mouse models enables mechanistic insights on how miRNAs regulate platelet protein content, but also platelet protein release. For example, miR-21 has recently been shown to attenuate the platelet release of transforming growth factor $\beta 1$, a master regulator of fibrosis. $^{2}$ In this issue, Garcia et al ${ }^{3}$ address the role of miR-126$3 p$, an important miRNA in platelets. While miR-126-3p is highly abundant in endothelial cells and was long considered endothelial specific, it is also expressed in megakaryocytes, and its potential role in platelet function has been overlooked. Garcia et $\mathrm{al}^{3}$ demonstrate that miR-126-3p is involved in the regulation of platelet activation using a relevant human cell type. The authors transfected CD34 + derived megakaryocytes with miR-126-3p and differentiated the megakaryocytes into platelet-like structures. Next, the authors performed platelet aggregation tests in a fibrinogen-coated flow chamber. They were able to show that miR-126-3p over-expression in megakaryocytes resulted in increased platelet activation. This is consistent with our previous findings in mice showing reduced platelet
Address for correspondence Manuel Mayr, MD, PhD, King's British Heart Foundation Centre, King's College London, 125 Coldharbour Lane, London SE5 NU, United Kingdom

(e-mail: manuel.mayr@kcl.ac.uk).

function upon treatment with a pharmacological inhibitor (antagomiR) against miR-126-3p. ${ }^{1}$ Moreover, the authors confirmed that miR-126-3p targets ADAM9 in megakaryocytes. ${ }^{1}$ ADAM9 has been shown to affect collagen-induced platelet aggregation. They also identified plexin-B2 as a new target of miR-126-3p. Plexin-B2 is involved in regulating platelet signalling. In summary, this article provides further evidence for a role of miR-126-3p in regulating platelet function. This finding should now be followed up in more detail using an appropriate model system.

\section{Conflict of Interest}

M. Mayr filed and licensed patent applications on miRNAs as biomarkers (EP15193448.6, EP2776580 B1, DE112013006129T5, GB2524692A, EP2576826 B, JP2013-513740).

\section{References}

1 Kaudewitz D, Skroblin P, Bender LH, et al. Association of microRNAs and YRNAs with platelet function. Circ Res 2016;118(03): 420-432

2 Barwari T, Eminaga S, Mayr U, et al. Inhibition of pro-fibrotic microRNA-21 affects platelets and their releasate. JCI Insights 2018;3:e123335

3 Garcia A, Dunoyer-Geindre S, Zapilko V, Nolli S, Reny J-L, Fontana P. Functional validation of microRNA-126-3p as a platelet reactivity regulator using human hematopoietic stem cells. Thromb Haemos 2019;119(02). Doi: 10.1055/s-0038-1676802 received

December 21, 2018

accepted

December 21, 2018 (c) 2019 Georg Thieme Verlag KG Stuttgart · New York
DOI https://doi.org/ 10.1055/s-0038-1677505. ISSN 0340-6245. 